Research Paper

\title{
Tendril extract of Cucurbita moschata suppresses NLRP3 inflammasome activation in murine macrophages and human trophoblast cells
}

\author{
Ji-Yeon Park ${ }^{1}$, Sung-Gang Jo ${ }^{1}$, Ha-Nul Lee ${ }^{2}$, Joo-Hee Choi ${ }^{1,3}$, Yeon-Ji Lee ${ }^{1}$, Young-Min Kim², Jeong-Yong \\ $\mathrm{Cho}^{2}$, Sung Ki Lee ${ }^{4}$, Jong-Hwan Park ${ }^{1,}{ }^{\otimes}$ \\ 1. Laboratory Animal Medicine, College of Veterinary Medicine and Animal Medical Institute, Chonnam National University, Gwangju, Republic of Korea \\ 2. Department of Food Science \& Technology, Chonnam National University, Gwangju, Republic of Korea \\ 3. Laboratory Animal Center, Daegu-Gyeongbuk Medical Innovation Foundation, Daegu, Republic of Korea \\ 4. Department of Obstetrics and Gynecology, College of Medicine, Konyang University, Daejeon, Republic of Korea \\ $\triangle$ Corresponding author: Jong-Hwan Park, DVM and Ph.D. Laboratory Animal Medicine, College of Veterinary Medicine, Chonnam National University, 77 \\ Yongbong-ro, Buk-gu, Gwangju 61186, Republic of Korea. Tel: +82-62-530-2834, E-mail address: jonpark@jnu.ac.kr \\ (C) The author(s). This is an open access article distributed under the terms of the Creative Commons Attribution License (https://creativecommons.org/licenses/by/4.0/). \\ See http://ivyspring.com/terms for full terms and conditions.
}

Received: 2019.08.04; Accepted: 2020.01.08; Published: 2020.04.06

\begin{abstract}
Inflammation is the root cause of many diseases that pose a serious threat to human health. Excessive inflammation can also result in preterm birth or miscarriage in pregnant women. Pumpkin (Cucurbita moschata Duchesne, CMD) is a well-known traditional health food and medicinal herb used in many countries to treat diabetes, obesity, osteoporosis, cancer and other diseases. In this study, we investigated the effects of hot water extract derived from the tendrils of $C$. moschata Duchesne (TCMD) on NLRP3 inflammasome activation in murine macrophages and human trophoblast cells. The TCMD treatment of LPS-primed bone marrow-derived macrophages (BMDMs) and human trophoblast cells attenuated NLRP3 inflammasome activation induced by inflammasome activators such as ATP, nigericin, and monosodium urate (MSU). TCMD treatment suppressed IL-1 $\beta$ secretion in a dose-dependent manner, without affecting IL-6 secretion. In addition, TCMD inhibited NLRP3-dependent pyroptosis in BMDMs. TCMD also suppressed the release of mature IL-1 $\beta$ and activation of cleaved-caspase- 1 via limited ASC oligomerization. Furthermore, TCMD significantly inhibited IL-1 $\beta$ secretion and pyroptotic cell death in human trophoblast cells. These results suggest that TCMD exhibits anti-inflammatory effects mediated via inhibition of NLRP3 inflammasome activation suggesting therapeutic potential against inflammatory diseases, preterm birth, and miscarriage.
\end{abstract}

Key words: tendril of Cucurbita moschata Duch, NLRP3 inflammasome,_bone marrow derived macrophage, trophoblast, SW.71

\section{Introduction}

Inflammation is one of the cellular defenses against deleterious stimuli such as tissue damage, infection, and exposure to toxic agents, and is strictly regulated by macrophages responsible for production of inflammatory molecules [1, 2]. In this systemic inflammatory response, macrophages produce inflammatory mediators such as NO, IL-6, TNF-a, and IL-1, which act as biological defenses in early infection [3]. However, persistent inflammatory response due to the overproduction of these mediators promotes mucosal injury, and as a result, leads to chronic inflammatory diseases such as arteriosclerosis, diabetes, arthritis, and cancer [4]. Intrauterine inflammation also causes spontaneous preterm birth and triggers various perinatal complications in premature infants including mortality [5]. However, therapeutic control of inflammation in preterm birth remains undeveloped. Control of intrauterine inflammatory response is an effective treatment strategy for prevention of preterm birth.

The NLRP3 inflammasome is the most studied inflammasome. The NLRP3 inflammasome is 
composed of NLRP3, caspase-1, and ASC, and assembled into an active protein complex in response to both microbial infection and endogenous "danger signal" [6-8]. The caspase- 1 is activated after the activation of the NLRP3 inflammasome. Activated caspase- 1 leads to cleavage of the precursor forms of the pro-IL-1 $\beta$, which mediate the inflammatory response [9-14]. However, excessive inflammation via NLRP3 activation results in serious diseases such as arthritis, asthma, inflammatory bowel disease (IBD), Parkinson's disease, Alzheimer's disease, and sepsis [15-22]. Preterm delivery is a major cause of neonatal death and is a life-threatening risk factor for the mother; however, the underlying pathophysiology and prevention is still not clear [23]. NLRP3 inflammasome activation induced by infection also causes preterm birth [24]. Activation of NLRP3 inflammasome mediates infection- or inflammation-induced parturition [23, 24]. In fact, many studies have shown that increased synthesis of IL-1 $\beta$ is associated with preterm delivery [25-29]. In view of the evidence that overexpression of NLRP3 inflammasome and the release of its products may contribute to disease, the pharmacological inhibition of NLRP3 inflammation may trigger certain diseases.

The use of dietary plants and herbal preparations as alternative medicines has recently received considerable attention worldwide [30]. Cucurbita moschata Duchesne (CMD), commonly known as "pumpkin", is one such plant that has been cultivated throughout the world and various parts of CMD have been used traditionally as functional food or medicine [30]. Studies reported that the fruit, fruit peel, stem, and seed extracts of CMD have anti-fatigue [31], wound-healing [32], anti-obesity [33], and anthelmintic [34] activities, respectively. Among them, dehydrodiconiferyl alcohol (DHCA), a lignan compound isolated from C. moschata, was suggested to exhibit anti-inflammatory effects by reducing $\mathrm{NO}$ production [35]. The tendril of CMD (TCMD) water extract and the purified active compound rutin showed anti-inflammatory effects by inhibiting the production of pro-inflammatory cytokines such as IL-6 and TNF-a [36]. However, the role of TCMD in regulating inflammasome activity is unknown. TCMD is one of the foods most pregnant women consumed traditionally in order to prevent spontaneous preterm birth because it alleviates swelling and prevents uterine contraction. However, there is no scientific basis for the effectiveness of TCMD in preventing preterm birth.

Regulation of IL-1 $\beta$ and inflammasome activity is important for effective treatment of inflammatory conditions including preterm birth. In this study, we thus investigated whether TCMD regulates NLRP3 inflammasome activation and IL-1 $\beta$ maturation in murine macrophages and human trophoblasts.

\section{Materials and Methods}

\section{Animals and ethics}

Wild type C57BL/6 male mice were purchased from Central Lab Animal Inc (Seoul, Korea). The animals were housed in the animal room at constant temperature $\left(22-24^{\circ} \mathrm{C}\right)$ and maintained under a strict $12 \mathrm{~h}$ lighting cycle. Mice were sacrificed by cervical dislocation, and macrophages were obtained from femur and tibia. The animal study protocols were approved by the Institutional Animal Care and Use Committee of Chonnam National University (Approval No. CNU IACUC-YB-R-2019-61).

\section{Preparation of TCMD extract}

Water extract of TCMD was prepared from dried tendrils of C. moschata Duch. TCMD (200 g) was added into $1 \mathrm{~L}$ of sterilized water and boiled at $90^{\circ} \mathrm{C}$ for $4 \mathrm{~h}$. This extract was filtered through filter papers (Whatman, Maidstone, UK), and concentrated using rotary evaporator (EYELA, Tokyo, Japan). The extract was lyophilized using a freeze dryer (Ilshin BioBase Co., Seoul, Korea) and stored at $-20^{\circ} \mathrm{C}$. The powder was dissolved in phosphate buffered saline (PBS) and diluted to the appropriate concentrations with culture medium.

\section{Reagents}

LPS, ATP, and monosodium urate crystals (MSU) were purchased from InvivoGen (San Diego, CA, USA). Nigericin sodium salt and rutin were purchased from Sigma-Aldrich (Sigma-Aldrich, St. Louis, MO, USA). Anti- $\beta$-actin antibody was obtained from Santa Cruz (Dallas, Texas, USA). Anti-IL-1 $\beta$, anti-caspase-1, anti-pro-IL-1 $\beta$, and anti-procaspase-1 were ordered from Cell Signaling Technology (Beverly, MA, USA).

\section{Preparation of murine macrophages and human trophoblast cells}

BMDMs derived from murine bone marrow were prepared as previously described [37]. Briefly, BMDMs were cultured in complete Iscove's modified Dulbecco's medium (IMDM, Gibco, Grand Island, NY, USA) including 30\% L929 cell culture supernatant, $10 \% \mathrm{FBS}, 1 \%$ sodium pyruvate, $1 \% \mathrm{MEM}$ Non-Essential Amino Acids (MEM NEAA) and 1\% penicillin/streptomycin in a $5 \% \mathrm{CO}_{2}$ incubator at $37^{\circ} \mathrm{C}$. After 3 days, $10 \mathrm{ml}$ of the fresh medium was added and the cells were incubated for an additional 2 days. The cells were seeded in 48 -well plates in triplicate at a concentration of $2 \times 10^{5}$ cells/well for 
cytokine analysis or in 6-well plates at a concentration of $2 \times 10^{6}$ cells / well for immunoblotting.

Sw.71 cells (a gift from Dr. Gil Mor, Yale University School of Medicine), representing a human trophoblast cell line, were cultured in DMEM (Welgene, Gyeongsangbuk-do, South Korea) containing 10\% FBS (Corning, Manassas, VA, USA), $100 \mathrm{U} / \mathrm{ml}$ penicillin-streptomycin (Gibco, Grand Island, NY, USA), 1 mM HEPES, 0.1 mM non-essential amino acids and $1 \mathrm{mM}$ sodium pyruvate. Cells were seeded in 48 -well $\left(5 \times 10^{4}\right.$ cells/well $)$ plates for cytokine analysis and in 6 -well $\left(5 \times 10^{5}\right.$ cells/well $)$ plates for real-time PCR analysis and incubated at $37^{\circ} \mathrm{C}$ and $5 \% \mathrm{CO}_{2}$ overnight.

\section{Inflammasome activation or inhibition}

BMDMs were layered on 48 -well plates at a concentration of $1 \times 10^{6}$ cells $/ \mathrm{ml}$ for cytokine analysis or on 6-well plates at a concentration of $1 \times 10^{6}$ cells $/ \mathrm{ml}$ for immunoblotting analysis. BMDMs were pretreated with various doses of TCMD for $2 \mathrm{~h}$ after priming with LPS (100 ng/ml) for $6 \mathrm{~h}$ and subsequently treated with ATP $(2 \mathrm{mM})$ or nigericin $(10 \mu \mathrm{M})$ for $30 \mathrm{~min}$ or monosodium urate (MSU) (200 $\mu \mathrm{g} / \mathrm{ml}$ ) for $4 \mathrm{~h}$. The culture supernatants were collected to measure the levels of IL-6 and IL-1 $\beta$. Sw.71 cells were plated in 48-well plates at a concentration of $5 \times 10^{5}$ cells $/ \mathrm{ml}$ for cytokine analysis or 6 -well plates at a concentration of $5 \times 10^{5}$ cells $/ \mathrm{ml}$ for Western blot.

\section{Measurement of cytokines}

The concentrations of IL- 6 and IL- $1 \beta$ in culture supernatants were measured using enzyme-linked immunosorbent assay (ELISA) kits (R\&D Systems, Minneapolis, MN, USA) according to the manufacturer's instructions.

\section{Western blotting analysis}

BMDMs and SW.71 cells were seeded into 6-well plates at a density of $1 \times 10^{6}$ cells $/ \mathrm{ml}$ and $5 \times 10^{5}$ cells $/ \mathrm{ml}$, respectively and incubated overnight. BMDMs were primed with LPS $(100 \mathrm{ng} / \mathrm{ml})$ for $6 \mathrm{~h}$ prior to ATP $(2 \mathrm{mM})$ or nigericin $(20 \mu \mathrm{M})$ for $30 \mathrm{~min}$ or MSU $(200 \mu \mathrm{g} / \mathrm{ml})$ for $4 \mathrm{~h}$. SW.71 cells were treated with MSU $(100 \mu \mathrm{g} / \mathrm{ml})$ for $72 \mathrm{~h}$. Cells were lysed at the indicated time point in a buffer containing $1 \%$ Nonidet P-40, $50 \mathrm{mM}$ Tris (pH 7.4), $250 \mathrm{mM} \mathrm{NaCl}, 5$ $\mathrm{mM}$ EDTA, $50 \mathrm{mM} \mathrm{NaF}, 1 \mathrm{mM} \mathrm{Na} 3 \mathrm{VO} 4$, and $0.02 \%$ NaN3 supplemented with protease inhibitor (complete, Mini, EDTA-free, Roche, Mannheim, Germany), phosphatase inhibitor (Phosphatase Inhibitor Cocktail 2, Sigma-Aldrich), and $2 \mathrm{mM}$ dithiothreitol. In an experiment using BMDMs, cell lysates and culture supernatant were used in combination. However, in the experiment using SW.71 cells, cell lysates were used for detection of tested proteins except cleaved IL-1 $\beta$. For the detection of cleaved IL-1 $\beta$, culture supernatant was used. Protein samples were separated by $10 \%, 12 \%$, and $15 \%$ sodium dodecyl sulfate polyacrylamide gel electrophoresis (SDS-PAGE) and transferred onto nitrocellulose membranes. The membranes were blocked with 5\% skim milk for $1 \mathrm{~h}$ at room temperature and probed with primary antibodies against pro-IL-1 $\beta$, IL-1 $\beta$, pro-caspase- 1 , caspase- 1 , and $\beta$-actin overnight at $4{ }^{\circ} \mathrm{C}$. The membranes were incubated with relevant secondary antibodies (Santa Cruz biotechnology) for $2 \mathrm{~h}$ at room temperature and proteins were detected using the ECL substrate (Bio-Rad, Hercules, CA, USA).

\section{Lactate dehydrogenase assay}

The release of lactate dehydrogenase (LDH) into the culture medium was determined using the LDH Cytotoxicity Assay Kit (Promega, Madison, WI) according to the manufacturer's instructions.

\section{ASC oligomerization assay}

After inflammasome activation in the absence or presence of TCMD extracts, the cells were harvested, resuspended in cold lysis buffer containing Triton X-100 and complete protease inhibitor cocktail (Roche, Mannheim, Germany), and passed 10 times through a 27 -gauge syringe. After centrifugation at $5000 \mathrm{rpm}$ for $10 \mathrm{~min}$, the supernatants (Triton-soluble fraction) were mixed with sample loading buffer $(5 \times)$ and used to detect ASC (Cell Signaling Technology, Cat No. 67824) and $\beta$-actin (Santa Cruz Biotechnology, sc-47778). The remaining cell pellets were resuspended in PBS buffer containing $2 \mathrm{mM}$ disuccinimidyl suberate (DSS, Sigma-Aldrich) cross-linker and were incubated at room temperature for $30 \mathrm{~min}$, followed by centrifugation at $5000 \mathrm{rpm}$ for $10 \mathrm{~min}$. To detect ASC oligomerization, the cross-linked pellets (Triton-insoluble fraction) were separated on $12 \%$ SDS-PAGE and transferred to NC membranes. These membranes were probed with primary antibodies against ASC. After immunoblotting with HRP-conjugated goat anti-rabbit IgG $(\mathrm{H}+\mathrm{L})$ (Invitrogen) or goat anti-mouse IgG $(\mathrm{H}+\mathrm{L})$ secondary antibodies (Invitrogen), the proteins were detected using Clarity Western ECL Substrate (Bio-Rad).

Cells were harvested after inflammasome activation with or without TCMD extract, resuspended in $0.5 \mathrm{ml}$ PBS buffer, and passed 10 times through a 27-gauge syringe. Cell lysates were centrifuged at $1000 \mathrm{rpm}$ for $10 \mathrm{~min}$ to collect the cells. 
A

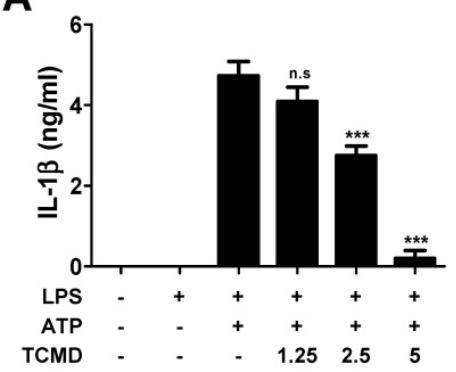

D
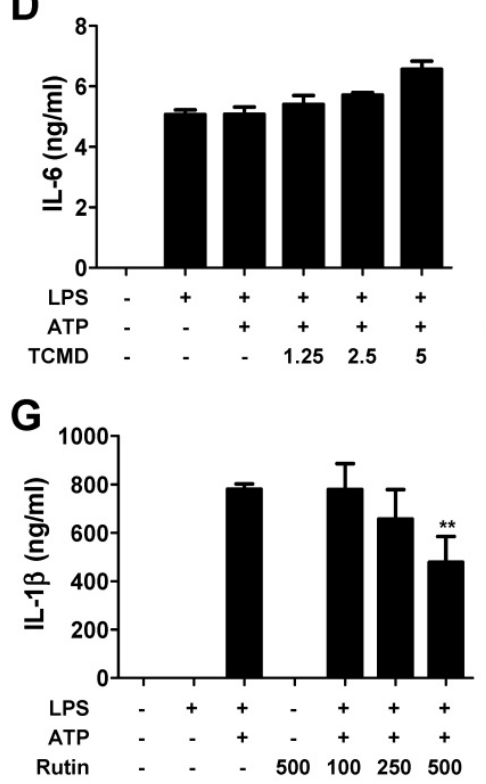

B

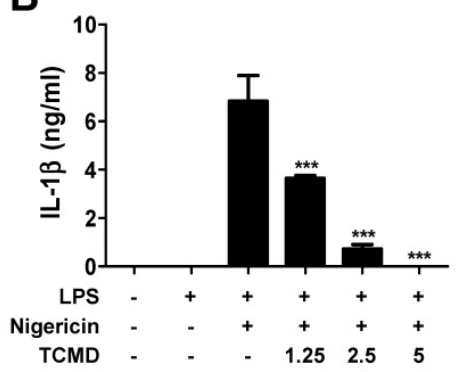

E

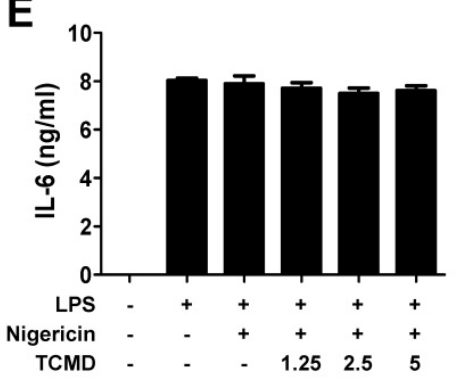

H

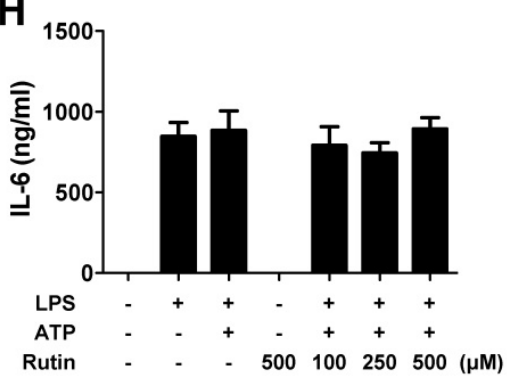

C

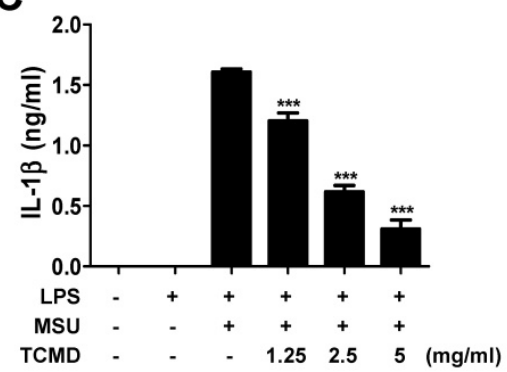

$\mathbf{F}$

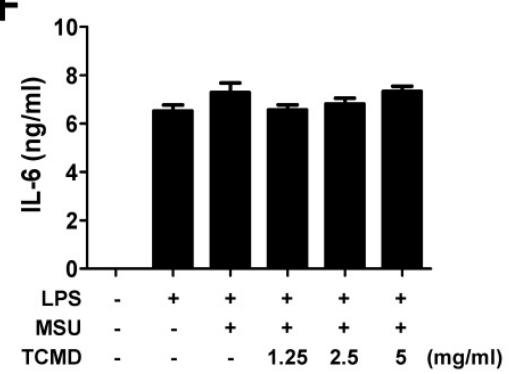

Figure 1. TCMD inhibits IL-1 $\beta$ secretion induced by NLRP3 inflammasome activation in BMDMs. BMDMs were primed with LPS (100 ng/ml) for $6 \mathrm{~h}$ prior to $\operatorname{TCMD}(1.25,2.5$, and $5 \mathrm{mg} / \mathrm{ml})(\mathrm{A}-\mathrm{F})$ or rutin $(100,250$, and $500 \mu \mathrm{M})(\mathrm{G}$ and $\mathrm{H})$ treatment for $2 \mathrm{~h}$, and subsequently treated with $2 \mathrm{mM}$ ATP for 30 min $(\mathrm{A}$ and $\mathrm{D})$, or $10 \mu \mathrm{M}$ nigericin for $30 \mathrm{~min}(\mathrm{~B}$ and $\mathrm{E})$, or $200 \mu \mathrm{g} / \mathrm{ml} \mathrm{MSU}$ for $4 \mathrm{~h}(\mathrm{C}$ and $\mathrm{F})$. The levels of IL-1 $\beta$ (A-C and G) and IL-6 (D-F and H) in culture supernatants were measured by ELISA. The results are from one experiment that is representative of three independent experiments and expressed as means $\pm S D$. $* * * P<0.001$.

Supernatants were diluted with equal volumes of lysis buffer containing triton X-100 and complete protease inhibitor cocktail (Roche, Mannheim, Germany), and centrifuged at $5000 \mathrm{rpm}$ for another 10 min to pellet the ASC pyroptosomes. The pellets were resuspended in PBS buffer containing $2 \mathrm{mM}$ disuccinimidyl suberate (DSS) cross-linker and were incubated at room temperature for $30 \mathrm{~min}$ followed by centrifugation at $5000 \mathrm{rpm}$ for $10 \mathrm{~min}$. The cross-linked pellets were fractionated on $12 \%$ SDS-PAGE, and ASC oligomerization was assessed by immunoblotting with ASC antibody (Cell signaling Technology; Cat No. 67824).

\section{Statistical analysis}

The statistical significance of differences between groups was determined via a two-tailed Student's t-test or one-way analysis of variance (ANOVA) followed by Bonferroni post hoc analysis (GraphPad Prism 5; GraphPad Software Inc., La Jolla, CA, USA). P-Values $<0.05$ were considered significant.

\section{Results}

TCMD inhibits inflammasome-induced IL-1 $\beta$ secretion in macrophages

We first investigated whether TCMD inhibited proinflammatory cytokines such as IL- 6 and IL- $1 \beta$. The IL-1 $\beta$ secretion was significantly increased in LPS-primed BMDMs in response to ATP, nigericin, and MSU and these increases were inhibited by TCMD in a dose-dependent manner (Figure 1A-1C). We investigated whether TCMD specifically inhibited IL-1 $\beta$ release by analyzing the release of another pro-inflammatory cytokine, IL- 6 , in the same culture supernatants. However, pre-treatment of cells with TCMD had no inhibitory effect on IL-6 release (Figure $1 \mathrm{D}-1 \mathrm{~F})$. A previous study revealed that rutin is a purified active compound in water extract of TCMD [36]. Accordingly, we further investigated whether rutin influences IL-1 $\beta$ secretion. As shown in Figure $1 \mathrm{G}$ and $1 \mathrm{H}$, rutin slightly decreased ATP-induced secretion of IL-1 $\beta$, but not IL-6, in LPS-primed 
BMDMs in a dose-dependent manner. These findings show that TCMD suppresses the secretion of IL- $1 \beta$ but not IL-6 in activated macrophages, indicating that TCMD specifically inhibits inflammasome response.

\section{TCMD inhibits pyroptosis by inhibiting LDH release in macrophages}

NLRP3 inflammasome activation also induces inflammatory cell death known as pyroptosis [38]. Pyroptotic cell death was assessed by measuring the LDH release after activating NLRP3 inflammasome in BMDMs and the effect of TCMD was evaluated. TCMD prevented ATP-induced LDH release in a dose-dependent manner in LPS-primed BMDMs (Figure 2A). Moreover, LDH release induced by nigericin and MSU was also inhibited by TCMD (Figure 2B, 2C). These results suggest that TCMD can suppress pyroptosis via inhibition of NLRP3 inflammasome activation.
TCMD inhibits cleavage of IL-1 $\beta$ and caspase- 1 in macrophages in response to ATP, nigericin, and MSU

Maturation of pro-IL-1 $\beta$ to induce inflammation requires caspase- 1 activation. Therefore, we evaluated whether TCMD regulates caspase- 1 activation and IL-1 $\beta$ maturation using Western blot analysis. The cleavage of IL-1 $\beta$ and caspase- 1 induced by ATP, nigericin, and MSU was significantly reduced by TCMD in the culture supernatants (Figure 3A-3C). Unlike the cleavage forms in the supernatants, the expression of pro-IL-1 $\beta$ and pro-caspase- 1 in the cell lysates was not changed by TCMD treatment (Figure $3 \mathrm{~A}-3 \mathrm{C})$. These results suggest that TCMD may regulates IL-1 $\beta$ secretion by inhibiting caspase-1 activation.
A

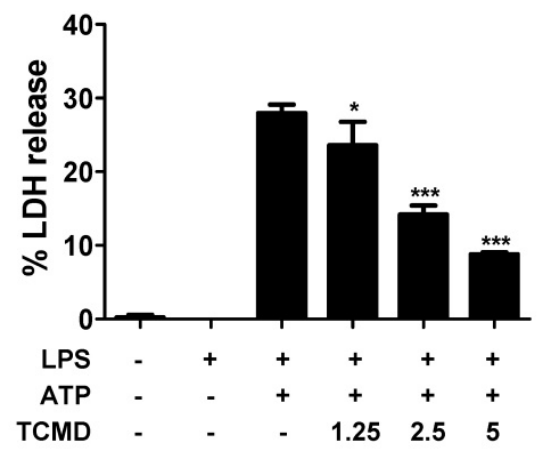

B

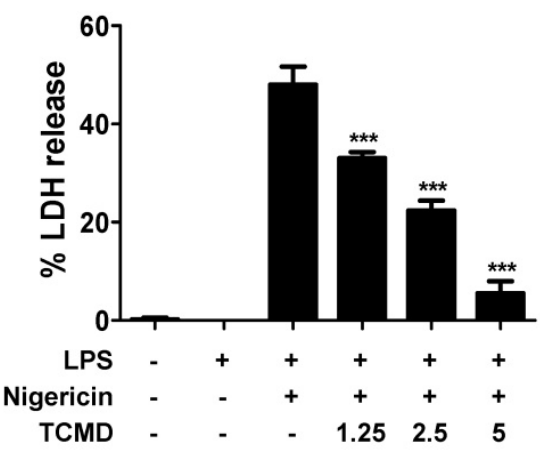

C

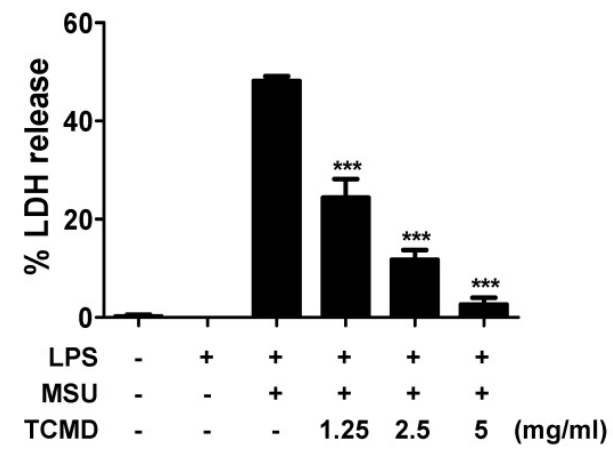

Figure 2. TCMD inhibits LDH release induced by NLRP3 inflammasome activation in BMDMs. BMDMs were primed with LPS (100 ng/ml) for $6 \mathrm{~h}$ prior to TCMD $(1.25,2.5$, and $5 \mathrm{mg} / \mathrm{ml})$ treatment for $2 \mathrm{~h}(\mathrm{~A}-\mathrm{C})$, and subsequently treated with $2 \mathrm{mM} \mathrm{ATP}$ for $30 \mathrm{~min}(\mathrm{~A})$, or $10 \mu \mathrm{M}$ nigericin for $30 \mathrm{~min}(\mathrm{~B})$, or $200 \mu \mathrm{g} / \mathrm{ml} \mathrm{MSU}$ for $4 \mathrm{~h}$ (C). The culture supernatants were collected for LDH determination. The results are from one experiment that is representative of three independent experiments and expressed as means \pm SD. $* * * P<0.001$

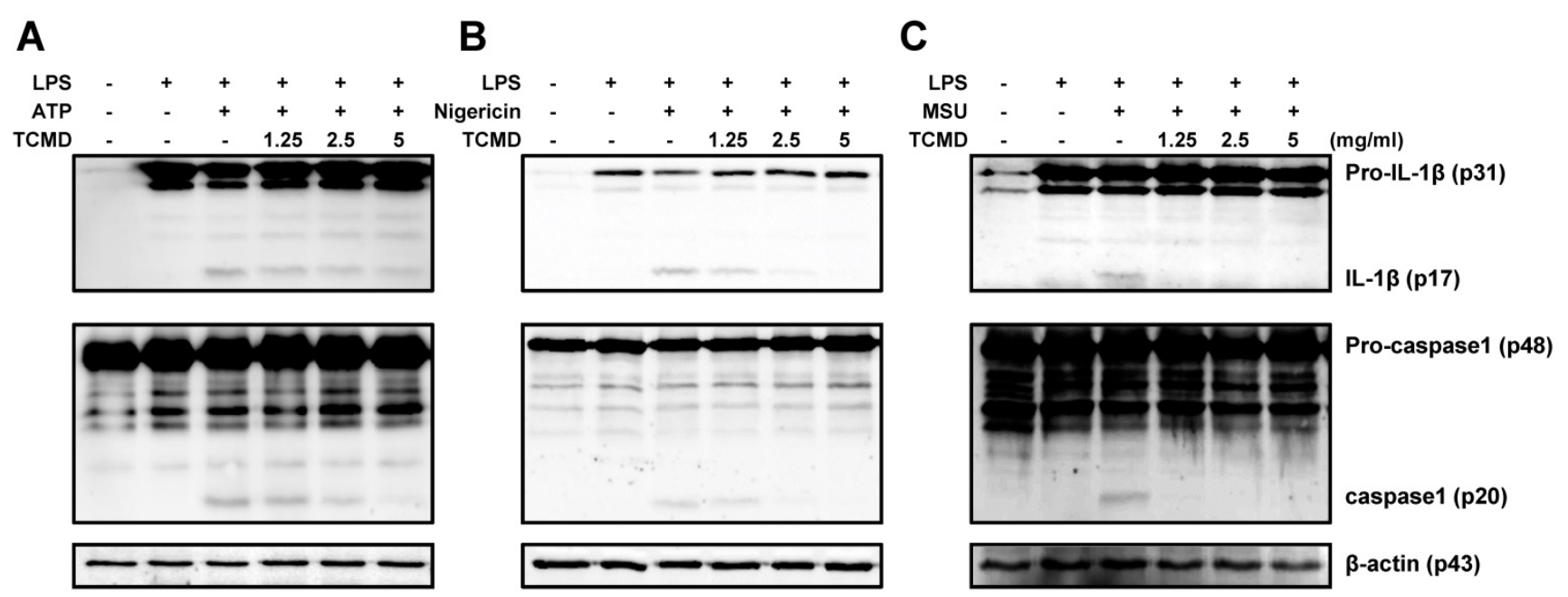

Figure 3. TCMD inhibits production of IL- $1 \beta$ and activation of caspase- 1 induced by NLRP3 inflammasome activation in BMDMs. BMDMs were primed with LPS $(100 \mathrm{ng} / \mathrm{ml})$ for $6 \mathrm{~h}$ prior to TCMD $(1.25,2.5$, and $5 \mathrm{mg} / \mathrm{ml})$ treatment for $2 \mathrm{~h}(\mathrm{~A}-\mathrm{C})$, and subsequently treated with $2 \mathrm{mM}$ ATP for $30 \mathrm{~min}(\mathrm{~A})$, or $10 \mu \mathrm{M}$ nigericin for $30 \mathrm{~min}$ (B), or $200 \mu \mathrm{g} / \mathrm{ml} \mathrm{MSU}$ for $4 \mathrm{~h}$ (C). Pro- and cleaved-forms of IL-1 $\beta$ and caspase-1 were detected by Western blot analysis. $\beta$-actin was used as a control for the loading volume. The results are from one experiment that is representative of two independent experiments. 


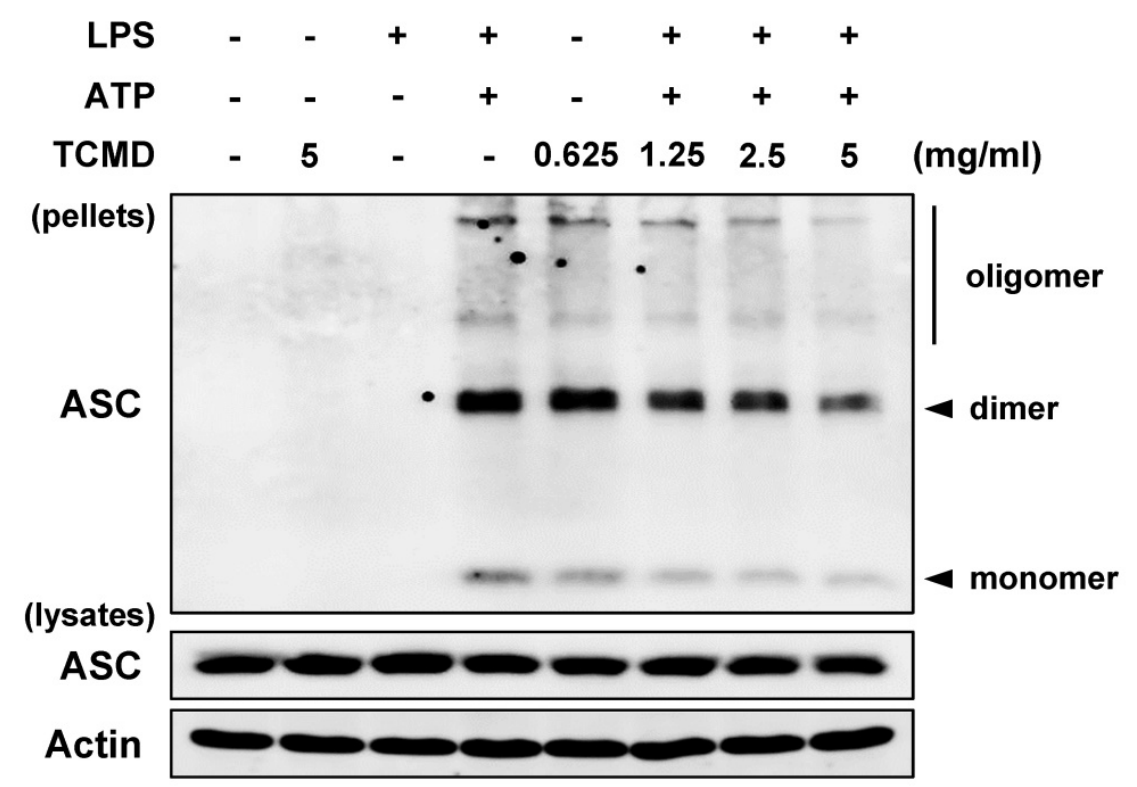

Figure 4. TCMD inhibits ASC oligomerization induced by NLRP3 inflammasome activation in BMDMs. BMDMs were primed with LPS (100 ng/ml) for $6 \mathrm{~h}$ prior to TCMD $(0.625,1.25,2.5$, and $5 \mathrm{mg} / \mathrm{ml})$ treatment for $2 \mathrm{~h}$, and subsequently treated with $2 \mathrm{mM}$ ATP for 30 min. ASC oligomerization was detected by Western blot analysis. $\beta$-actin was used as a control for the loading volume. The results are from one experiment that is representative of two independent experiments.

\section{TCMD inhibits the oligomerization of ASC in macrophages}

A previous study reported that IL-1 $\beta$ secretion is induced by activated caspase- 1 via assembly of an inflammasome complex composed of NLRP3, oligomerized ASC, and caspase-1 [39]. Moreover, ASCs form a dimer or oligomer in inflammasomeactivated conditions [40]. We thus examined the impact of TCMD on ASC oligomerization in BMDMs, which are regarded as the hallmarks of inflammasome formation. Western blot analysis revealed that ASC oligomerization induced by ATP was decreased by TCMD treatment in a dose-dependent manner (Figure 4), suggesting that TCMD has the potential to inhibit inflammasome activation via regulation of ASC oligomerization.

\section{TCMD inhibits IL- $1 \beta$ secretion and LDH release in human trophoblasts}

In a dose-dependent experiment, we found that ATP treatment did not induce IL-1 $\beta$ secretion in SW.71 cells at ranges of $5-500 \mu \mathrm{M}$ and nigericin could induce only small amount secretion of IL-1 $\beta$ at doses of 0.5 and $5 \mu \mathrm{M}$ (Figure 5A, 5B). In contrast, MSU (100 $\mu \mathrm{g} / \mathrm{ml}$ ) could produce huge amount of IL- $1 \beta$ in the cells, even without LPS priming (Figure 5A, 5B), which is consistent with a previous study [41]. To determine whether TCMD inhibits IL- $1 \beta$ secretion in human trophoblasts, we administered TCMD prior to MSU activation and measured the release of IL-1 $\beta$ by ELISA and Western blot. The MSU-stimulated IL-1 $\beta$ secretion was significantly down-regulated by TCMD pre-treatment in a dose-dependent manner (Figure
5C). In addition, cleaved caspase- 1 and IL- $1 \beta$ was also reduced by TCMD in SW.71 cells in response to MSU (Figure 5D). TCMD treatment also decreased LDH release and improved cell viability (Figure 5E, 5F). Taken together, TCMD inhibits NLRP3 inflammasome activity and pyroptotic cell death in human trophoblasts.

\section{Discussion}

Inflammasome activity is important for the maturation and secretion of IL-1 $\beta$, which is involved in the pathophysiology of tissue damage [42-46]. Increased levels of IL-1 $\beta$ have a detrimental effect on pregnancy, leading to complications such as chorioamnionitis, preeclampsia, and preterm birth [47-50]. CMD has been widely used in traditional herbal remedies and foods and has received considerable attention due to its extensive bioactivity as an anti-diabetic [51], anti-cancer [52], anti-obesity [53], and anti-inflammatory agent [35]. However, the efficacy of TCMD has not been fully understood. In the present study, we found that TCMD plays a crucial role in inhibiting NLRP3 inflammasome activation in murine macrophages and human trophoblast cells. We suggest that TCMD is a potential food and therapeutic agent in the prevention of inflammatory conditions including preterm labor.

Our results showed that TCMD pretreatment significantly inhibited IL-1 $\beta$ synthesis by ATP, nigericin, and MSU in LPS-primed BMDMs. However, TCMD had no effect on the release of IL-6, which indicates that TCMD specifically regulates NLRP3 inflammasome activation. In addition to the 
A
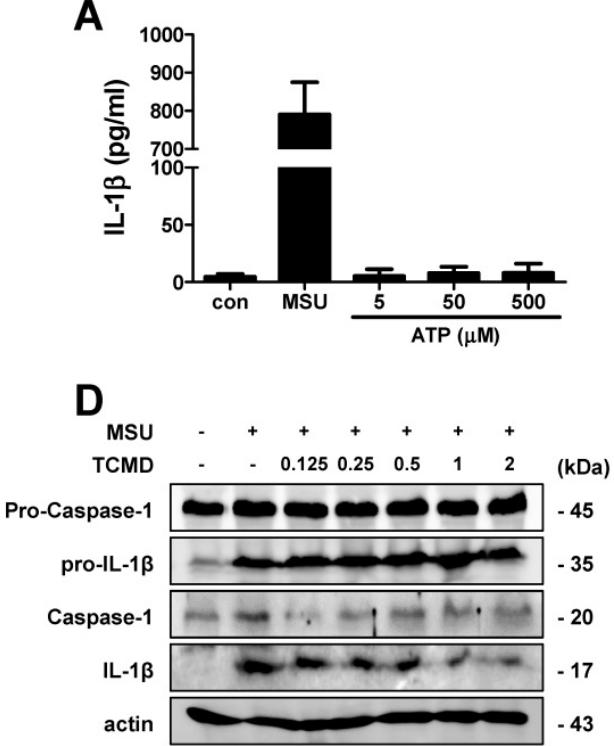

B

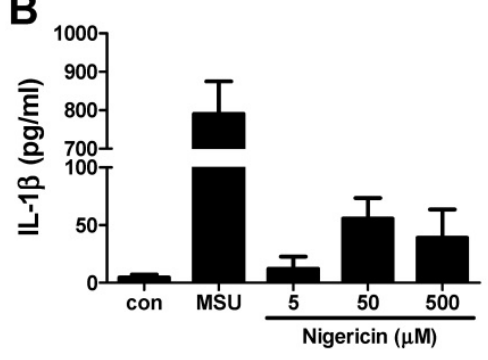

E

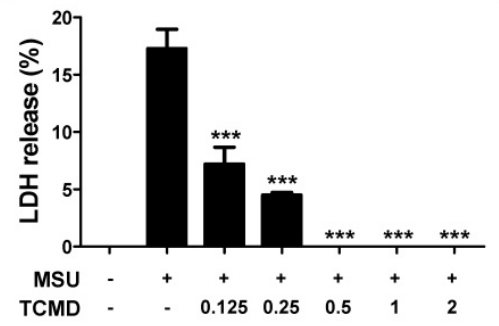

C

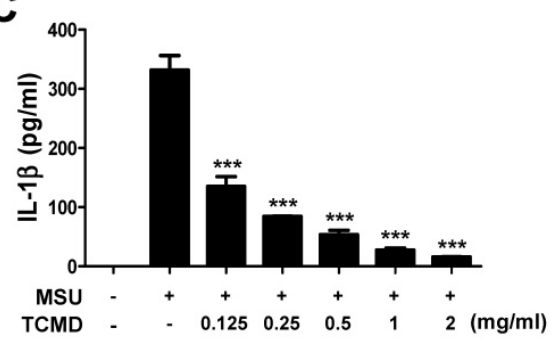

F

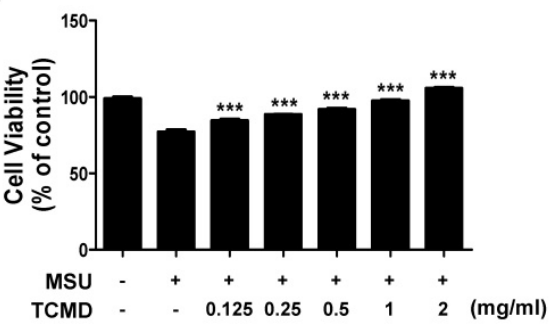

Figure 5. TCMD inhibits IL- $1 \beta$ secretion and LDH release induced by NLRP3 inflammasome activation in human trophoblasts. SW.71 cells were treated with ATP $(5-500 \mu \mathrm{M})$, nigericin $(0.05-5 \mu \mathrm{M})$, and MSU $(100 \mu \mathrm{g} / \mathrm{ml})$ for $72 \mathrm{~h}(\mathrm{~A}$ and $\mathrm{B})$. The cells were pretreated with TCMD $(0.125,0.25,0.5,1$, and $2 \mathrm{mg} / \mathrm{ml})$ for $2 \mathrm{~h}$ and activated with MSU $(100 \mathrm{\mu g} / \mathrm{ml})$ for $72 \mathrm{~h}(\mathrm{~A}-\mathrm{F})$. The levels of IL-1 $\beta$ in culture supernatants were measured by ELISA (A-C). Pro- and cleaved-forms of IL-1 $\beta$ were detected by western blot analysis (D). LDH release was measured in culture supernatants $(E)$. Cell viability was measured by MTT assay (F). $\beta$-actin was used as a control for the loading volume. The results are from one experiment that is representative of two (D) or three (A-C, E, F) independent experiments and expressed as means $\pm S D$. $* P<0.05, * * P<0.01, * * * P<0.001$.

secretion and maturation of IL-1 $\beta$, the inflammasome activation triggers programmed, pro-inflammatory cell death known as pyroptosis [20]. Pyroptosis is mediated via caspase-1 activation inducing the formation of membrane pores, cellular swelling followed by membrane destruction, and release of intracellular content into the extracellular space, including cytoplasmic proteins such as LDH [54]. Our results indicated that TCMD inhibits $\mathrm{LDH}$ release, suggesting that TCMD can potentially prevent pyroptotic cell death in macrophages.

Many ASC molecular events related to inflammasome activation have been reported [55]. The adapter protein ASC is essential for both NLRP3 and AIM2 inflammasome formation [56, 57]. Therefore, investigation of ASC oligomerization is regarded as a hallmark of inflammasome formation [55]. In a previous study, Artemisia princeps extract (APO) inhibited ASC oligomerization triggered by ATP-, silica-, or nigericin-induced NLRP3 inflammasomes and poly (dA:dT)-induced AIM2 inflammasome activation [55]. Consistent with that study, our results indicate that the ASC oligomerization was increased in the LPS-primed BMDMs activated by ATP and was dose-dependently inhibited by TCMD (Figure 4). These results indicate that TCMD may suppress inflammatory activation via regulation of ASC oligomerization.

In the presence of hypertension, the risk of preterm birth was increased with increasing uric acid levels [58]. Women with hypertension and hyperuricemia had a 3.6-week shorter gestation period and a 7-fold higher prevalence of premature birth than women with hypertension alone [58]. Therefore, an increase in uric acid level during pregnancy may induce preterm birth. Preterm birth is also associated with placental infection and trophoblast inflammation [24]. In trophoblasts, NLRP3 inflammasome activation and elevated levels of proinflammatory cytokines including IL-1 $\beta$ have been associated with pregnancy complications, such as preeclampsia, miscarriage, and preterm birth [23, 26]. Therefore, the regulation of intra-placental inflammation via extracellular or intracellular stimulation may prevent preterm labor and stillbirth. Because first trimester trophoblast cells express pattern recognition receptors (PRRs) such as TLRs and Nod proteins, stimulation by bacteria and viruses induces inflammatory response [41]. A previous study demonstrated that treatment with antiphospholipid antibodies (aPL) via TLR4 activation raises the uric acid levels in human trophoblasts, which in turn activate the Nalp3/ASC inflammasome inducing IL-1 $\beta$ secretion [26]. This mechanism may trigger placental dysfunction and exacerbate the pregnancy outcome of patients with antiphospholipid syndrome (APS) [26]. Untreated first trimester trophoblast cells express pro-IL-1 $\beta$ at a high level and therefore MSU acting as DAMP to activate NLRP3 inflammasome, was treated with SW.71 cells [23, 26, 41]. Several studies have demonstrated that MSU induces IL-1 $\beta$ secretion by activating inflammasome in trophoblasts [24, 41]. Our results consistently showed that MSU treatment induces IL-1 $\beta$ secretion 
in SW.71 cells, which was significantly inhibited by TCMD. TCMD also inhibits MSU-induced LDH release and restores cell viability in SW.71 cells.

Overall, TCMD exerts anti-inflammatory effects by inhibiting IL-1 $\beta$ secretion in BMDMs and human trophoblast cells. However, TCMD had no effect on the expression of pro-IL-1 $\beta$, and procaspase- 1 , and ASC, which indicates that TCMD specifically inhibits inflammasome activation. These results indicate that TCMD acts as a direct inhibitor of the NLRP3 inflammasome. This report is the first of its kind to provide scientific evidence suggesting that TCMD is a highly effective natural extract that can be used to modulate the inflammatory response via inhibition of NLRP3 inflammasome activation.

In conclusion, our present data indicate that TCMD is a potential anti-inflammatory agent that can be developed further for the treatment of inflammatory disorders and preterm birth. Therefore, additional in vivo studies are needed to further confirm the efficacy of TCMD in pregnant women and fetuses.

\section{Abbreviations}

CMD: Cucurbita moschata Duchesne; TCMD: tendrils of Cucurbita moschata Duchesne; BMDMs: bone marrow-derived macrophages; MSU: monosodium urate; IBD: inflammatory bowel disease; DHCA: dehydrodiconiferyl alcohol; PBS: phosphate buffered saline; MEM NEAA: MEM Non-Essential Amino Acids; ELISA: enzyme-linked immunosorbent assay; LDH: lactate dehydrogenase; DSS: disuccinimidyl suberate; APO: Artemisia princeps extract; PRRs: pattern recognition receptors; aPL: antiphospholipid antibodies; APS: antiphospholipid syndrome.

\section{Acknowledgments}

This study was supported by a grant (HI17C1238) from the Korean Health Technology R\&D Project funded by the Ministry of Health \& Welfare, Republic of Korea.

\section{Competing Interests}

The authors have declared that no competing interest exists.

\section{References}

1. Dia VP, Bradwell J, Pangloli P. Sorghum Phenolics Inhibits Inflammasomes in Lipopolysaccharide (LPS)-Primed and Adenosine Triphosphate (ATP)-Activated Macrophages. Plant foods for human nutrition. 2019; 74: 307-315

2. Han JW, Shim DW, Shim EJ, Kim MK, Shin YK, Kwak SB, et al. Syneilesis palmata (Thunb.) Maxim. extract attenuates inflammatory responses via the regulation of TRIF-dependent signaling and inflammasome activation. Journal of ethnopharmacology. 2015; 166: 1-4.

3. Higuchi M, Higashi N, Taki H, Osawa T. Cytolytic mechanisms of activated macrophages. Tumor necrosis factor and L-arginine-dependent mechanisms act synergistically as the major cytolytic mechanisms of activated macrophages. Journal of immunology. 1990; 144: 1425-31.

4. Ljung T, Lundberg S, Varsanyi M, Johansson C, Schmidt PT, Herulf M, et al. Rectal nitric oxide as biomarker in the treatment of inflammatory bowel disease: responders versus nonresponders. World journal of gastroenterology. 2006; 12: 3386-92.

5. Cappelletti M, Della Bella S, Ferrazzi E, Mavilio D, Divanovic S. Inflammation and preterm birth. Journal of leukocyte biology. 2016; 99: 67-78.

6. Zhao X, Pu D, Zhao Z, Zhu H, Li H, Shen Y, et al. Teuvincenone F Suppresses LPS-Induced Inflammation and NLRP3 Inflammasome Activation by Attenuating NEMO Ubiquitination. Frontiers in pharmacology. 2017; 8: 565.

7. Martinon F, Mayor A, Tschopp J. The inflammasomes: guardians of the body. Annual review of immunology. 2009; 27: 229-65.

8. Davis BK, Wen H, Ting JP. The inflammasome NLRs in immunity, inflammation, and associated diseases. Annual review of immunology. 2011; 29: 707-35.

9. Zhang $\mathrm{H}$, Chen $\mathrm{S}$, Zeng $\mathrm{M}$, Lin $\mathrm{D}$, Wang $\mathrm{Y}$, Wen $\mathrm{X}$, et al Apelin-13 Administration Protects Against LPS-Induced Acute Lung Injury by Inhibiting NF-kappaB Pathway and NLRP3 Inflammasome Activation. Cellular physiology and biochemistry : international journal of experimental cellular physiology, biochemistry, and pharmacology. 2018; 49: 1918-32.

10. Hornung V, Bauernfeind F, Halle A, Samstad EO, Kono H, Rock KL, et al. Silica crystals and aluminum salts activate the NALP3 inflammasome through phagosomal destabilization. Nature immunology. 2008; 9: 847-56.

11. Kailasan Vanaja S, Rathinam VA, Atianand MK, Kalantari P, Skehan B, Fitzgerald KA, et al. Bacterial RNA:DNA hybrids are activators of the NLRP3 inflammasome. Proceedings of the National Academy of Sciences of the United States of America. 2014: 111: 7765-70.

12. Rathinam VA, Vanaja SK, Waggoner L, Sokolovska A, Becker C, Stuart LM, et al. TRIF licenses caspase-11-dependent NLRP3 inflammasome activation by gram-negative bacteria. Cell. 2012; 150: 606-19.

13. Willingham SB, Allen IC, Bergstralh DT, Brickey WJ, Huang MT, Taxman DJ, et al. NLRP3 (NALP3, Cryopyrin) facilitates in vivo caspase-1 activation, necrosis, and HMGB1 release via inflammasome-dependent and -independent pathways. Journal of immunology. 2009; 183: 2008-15.

14. van de Veerdonk FL, Netea MG, Dinarello CA, Joosten LA. Inflammasome activation and IL-1beta and IL-18 processing during infection. Trends in immunology. 2011; 32: 110-6.

15. Ko W, Quang TH, Sohn JH, Yim JH, Kang DG, Lee HS, et al. Anti-inflammatory effect of 3,7-dimethyl-1,8-hydroxy-6-methoxyisochroman via nuclear factor erythroid 2-like 2-mediated heme oxygenase-1 expression in lipopolysaccharide-stimulated RAW264.7 and BV2 cells. Immunopharmacology and immunotoxicology. 2019; 41: 337-48.

16. Lucas K, Maes M. Role of the Toll Like receptor (TLR) radical cycle in chronic inflammation: possible treatments targeting the TLR4 pathway. Molecular neurobiology. 2013; 48: 190-204

17. Guo C, Fu R, Wang S, Huang Y, Li X, Zhou M, et al. NLRP3 inflammasome activation contributes to the pathogenesis of rheumatoid arthritis. Clinical and experimental immunology. 2018; 194: 231-43.

18. Kim RY, Pinkerton JW, Essilfie AT, Robertson AAB, Baines KJ, Brown AC, et al. Role for NLRP3 Inflammasome-mediated, IL-1beta-Dependent Responses in Severe, Steroid-Resistant Asthma. American journal of respiratory and critical care medicine. 2017; 196: 283-97.

19. Zhen $Y$, Zhang H. NLRP3 Inflammasome and Inflammatory Bowel Disease. Frontiers in immunology. 2019; 10: 276.

20. Wang $\mathrm{S}$, Yuan $\mathrm{YH}$, Chen NH, Wang HB. The mechanisms of NLRP3 inflammasome/pyroptosis activation and their role in Parkinson's disease. International immunopharmacology. 2019; 67: 458-64.

21. Tan MS, Yu JT, Jiang $T$, Zhu XC, Tan L. The NLRP3 inflammasome in Alzheimer's disease. Molecular neurobiology. 2013; 48: 875-82.

22. Lee S, Nakahira K, Dalli J, Siempos II, Norris PC, Colas RA, et al. NLRP3 Inflammasome Deficiency Protects against Microbial Sepsis via Increased Lipoxin B4 Synthesis. American journal of respiratory and critical care medicine. 2017; 196: 713-26.

23. Tamura K, Ishikawa G, Yoshie M, Ohneda W, Nakai A, Takeshita T, et al Glibenclamide inhibits NLRP3 inflammasome-mediated IL-1beta secretion in human trophoblasts. Journal of pharmacological sciences. 2017; 135: 89-95.

24. Chen CY, Chen CY, Liu CC, Chen CP. Omega-3 polyunsaturated fatty acids reduce preterm labor by inhibiting trophoblast cathepsin $\mathrm{S}$ and inflammasome activation. Clinical science. 2018; 132: 2221-39.

25. Skogstrand K, Hougaard DM, Schendel DE, Bent NP, Svaerke C, Thorsen P. Association of preterm birth with sustained postnatal inflammatory response. Obstetrics and gynecology. 2008; 111: 1118-28.

26. Mulla MJ, Salmon JE, Chamley LW, Brosens JJ, Boeras CM, Kavathas PB, et al. A role for uric acid and the Nalp3 inflammasome in antiphospholipid antibody-induced IL-1beta production by human first trimester trophoblast. PloS one. 2013; 8: e65237.

27. Hirsch E, Blanchard R, Mehta SP. Differential fetal and maternal contributions to the cytokine milieu in a murine model of infection-induced preterm birth. American journal of obstetrics and gynecology. 1999; 180: 429-34.

28. Rinehart BK, Terrone DA, Lagoo-Deenadayalan S, Barber WH, Hale EA, Martin JN, Jr., et al. Expression of the placental cytokines tumor necrosis factor alpha, interleukin 1beta, and interleukin 10 is increased in preeclampsia. American journal of obstetrics and gynecology. 1999; 181: 915-20. 
29. Munno I, Chiechi LM, Lacedra G, Putignano G, Patimo C, Lobascio A, et al. Spontaneous and induced release of prostaglandins, interleukin (IL)-1beta, IL-6, and tumor necrosis factor-alpha by placental tissue from normal and preeclamptic pregnancies. American journal of reproductive immunology. 1999; 42: 369-74

30. Caili F, Huan S, Quanhong L. A review on pharmacological activities and utilization technologies of pumpkin. Plant foods for human nutrition. 2006; 61: 73-80.

31. Wang SY, Huang WC, Liu CC, Wang MF, Ho CS, Huang WP, et al. Pumpkin (Cucurbita moschata) fruit extract improves physical fatigue and exercise performance in mice. Molecules. 2012; 17: 11864-76.

32. Bahramsoltani R, Farzaei MH, Abdolghaffari AH, Rahimi R, Samadi N, Heidari $\mathrm{M}$, et al. Evaluation of phytochemicals, antioxidant and burn wound healing activities of Cucurbita moschata Duchesne fruit peel. Iranian journal of basic medical sciences. 2017; 20: 798-805.

33. Choi H, Eo H, Park K, Jin M, Park EJ, Kim SH, et al. A water-soluble extract from Cucurbita moschata shows anti-obesity effects by controlling lipid metabolism in a high fat diet-induced obesity mouse model. Biochemical and biophysical research communications. 2007; 359: 419-25.

34. Grzybek M, Kukula-Koch W, Strachecka A, Jaworska A, Phiri AM, Paleolog J, et al. Evaluation of Anthelmintic Activity and Composition of Pumpkin (Cucurbita pepo L.) Seed Extracts-In Vitro and in Vivo Studies. International journal of molecular sciences. 2016; 17

35. Lee J, Kim S. Upregulation of heme oxygenase-1 expression by dehydrodiconiferyl alcohol (DHCA) through the AMPK-Nrf2 dependent pathway. Toxicology and applied pharmacology. 2014; 281: 87-100.

36. Jeong $\mathrm{HN}$, Choi JH, Lee HN, Lee $\mathrm{SH}$, Cho SC, Park JH, et al. Inflammation inhibitory effect of water extract from pumpkin's tendril. The Korean Society of Food Preservation. 2017; 24: 1122-1128.

37. Celada A, Gray PW, Rinderknecht E, Schreiber RD. Evidence for a gamma-interferon receptor that regulates macrophage tumoricidal activity. The Journal of experimental medicine. 1984; 160: 55-74.

38. He WT, Wan H, Hu L, Chen P, Wang X, Huang Z, et al. Gasdermin D is an executor of pyroptosis and required for interleukin-1beta secretion. Cell research. 2015; 25: 1285-98.

39. Yu JW, Wu J, Zhang Z, Datta P, Ibrahimi I, Taniguchi S, et al. Cryopyrin and pyrin activate caspase-1, but not NF-kappaB, via ASC oligomerization. Cell death and differentiation. 2006; 13: 236-49.

40. Fernandes-Alnemri T, Yu JW, Juliana C, Solorzano L, Kang S, Wu J, et al. The AIM2 inflammasome is critical for innate immunity to Francisella tularensis. Nature immunology. 2010; 11: 385-93.

41. Mulla MJ, Myrtolli K, Potter J, Boeras C, Kavathas PB, Sfakianaki AK, et al. Uric acid induces trophoblast IL-1beta production via the inflammasome: implications for the pathogenesis of preeclampsia. American journal of reproductive immunology. 2011; 65: 542-8

42. Primiano MJ, Lefker BA, Bowman MR, Bree AG, Hubeau C, Bonin PD, et al. Efficacy and Pharmacology of the NLRP3 Inflammasome Inhibitor CP-456,773 (CRID3) in Murine Models of Dermal and Pulmonary Inflammation. Journal of immunology. 2016; 197: 2421-33.

43. Guo H, Callaway JB, Ting JP. Inflammasomes: mechanism of action, role in disease, and therapeutics. Nature medicine. 2015; 21: 677-87.

44. Masters SL, Latz E, O'Neill LA. The inflammasome in atherosclerosis and type 2 diabetes. Science translational medicine. 2011; 3: 81ps17.

45. Dinarello CA, Simon A, van der Meer JW. Treating inflammation by blocking interleukin-1 in a broad spectrum of diseases. Nature reviews Drug discovery. 2012; 11: 633-52.

46. Iyer SS, Pulskens WP, Sadler JJ, Butter LM, Teske GJ, Ulland TK, et al. Necrotic cells trigger a sterile inflammatory response through the Nlrp3 inflammasome. Proceedings of the National Academy of Sciences of the United States of America. 2009; 106: 20388-93.

47. Nadeau-Vallee M, Quiniou C, Palacios J, Hou X, Erfani A, Madaan A, et al. Novel Noncompetitive IL-1 Receptor-Biased Ligand Prevents Infection- and Inflammation-Induced Preterm Birth. Journal of immunology. 2015; 195: 3402-15.

48. Whidbey C, Vornhagen J, Gendrin C, Boldenow E, Samson JM, Doering K, et al. A streptococcal lipid toxin induces membrane permeabilization and pyroptosis leading to fetal injury. EMBO molecular medicine. 2015; 7: 488-505.

49. Flores-Herrera H, Garcia-Lopez G, Diaz NF, Molina-Hernandez A, Osorio-Caballero M, Soriano-Becerril D, et al. An experimental mixed bacterial infection induced differential secretion of proinflammatory cytokines (IL-1beta, TNFalpha) and proMMP-9 in human fetal membranes. Placenta. 2012; 33: 271-7.

50. Jaiswal MK, Agrawal V, Mallers T, Gilman-Sachs A, Hirsch E, Beaman KD. Regulation of apoptosis and innate immune stimuli in inflammation-induced preterm labor. Journal of immunology. 2013; 191: 5702-13.

51. Jiang $\mathrm{Z}$, Du $\mathrm{O}$. Glucose-lowering activity of novel tetrasaccharide glyceroglycolipids from the fruits of Cucurbita moschata. Bioorganic \& medicinal chemistry letters. 2011; 21: 1001-3.

52. Zhang $\mathrm{B}$, Huang $\mathrm{H}$, Xie $\mathrm{J}, \mathrm{Xu} \mathrm{C}$, Chen $\mathrm{M}$, Wang $\mathrm{C}$, et al. Cucurmosin induces apoptosis of BxPC-3 human pancreatic cancer cells via inactivation of the EGFR signaling pathway. Oncology reports. 2012; 27: 891-7.

53. Lee J, Kim D, Choi J, Choi H, Ryu JH, Jeong J, et al. Dehydrodiconiferyl alcohol isolated from Cucurbita moschata shows anti-adipogenic and anti-lipogenic effects in 3T3-L1 cells and primary mouse embryonic fibroblasts. The Journal of biological chemistry. 2012; 287: 8839-51.
54. Rayamajhi M, Zhang Y, Miao EA. Detection of pyroptosis by measuring released lactate dehydrogenase activity. Methods in molecular biology. 2013; 1040: 85-90.

55. Kwak SB, Koppula S, In EJ, Sun X, Kim YK, Kim MK, et al. Artemisia Extract Suppresses NLRP3 and AIM2 Inflammasome Activation by Inhibition of ASC Phosphorylation. Mediators of inflammation. 2018; 2018: 6054069.

56. Coll RC, Robertson A, Butler M, Cooper M, O'Neill LA. The cytokine release inhibitory drug CRID3 targets ASC oligomerisation in the NLRP3 and AIM2 inflammasomes. PloS one. 2011; 6: e29539.

57. Lopez-Castejon G, Luheshi NM, Compan V, High S, Whitehead RC, Flitsch S, et al. Deubiquitinases regulate the activity of caspase-1 and interleukin-1beta secretion via assembly of the inflammasome. The Journal of biological chemistry. 2013; 288: 2721-33.

58. Roberts JM, Bodnar LM, Lain KY, Hubel CA, Markovic N, Ness RB, et al. Uric acid is as important as proteinuria in identifying fetal risk in women with gestational hypertension. Hypertension. 2005; 46: 1263-9. 